\title{
ELECTROMYOSTIMULATION AND PLYOMETRIC TRAINING EFFECTS ON JUMPING AND SPRINT TIME
}

\author{
J. AZAEL HERRERO ${ }^{1}$, MIKEL IZQUIERDO ${ }^{2,3}$, NICOLA A. MAFFIULETTI ${ }^{4}$, JUAN \\ GARCÍA-LÓPEZ ${ }^{2}$
}

\begin{abstract}
${ }^{I}$ Faculty of Health Sciences. European University Miguel de Cervantes, Valladolid, SPAIN ${ }^{2}$ Physical Activity and Sport Sciences Faculty. University of León. SPAIN; ${ }^{3}$ Studies, Research and Sports Medicine Center, Government of Navarra, SPAIN; ${ }^{4}$ Faculté des Sciences du Sport de Dijon, Université de Bourgogne, Dijon, FRANCE.
\end{abstract}

Running head: EMS, sprint time and jumping

Corresponding author: Dr. Mikel Izquierdo Centro de Estudios, Investigación y Medicina del Deporte de Navarra. Instituto Navarro de Deporte y Juventud. Gobierno de Navarra.

$\mathrm{C} /$ Sangüesa 34

31005 Pamplona (Navarra)

Spain

Fax: + 34948292636

Email: mizquierdo@unileon.es 
ABSTRACT

Purpose: This study compared the effects of four week training periods of electromyostimulation (EMS), plyometric training (P), or combined EMS and P training of the knee extensor muscles on $20 \mathrm{~m}$ sprint time (ST), jumping ability (SJ and CMJ), maximal isometric strength (MVC) and muscle cross-sectional area (CSA). Methods: Forty subjects were randomly assigned to one of the four treatment groups: Electromyostimulation (EG); plyometric (PG); combined EMG and P (EPG); and a control group (CG) that performed 4 times per week. Subjects were tested before and after the training program, as well as once more after 2 wk of detraining. Results: A significant increase $(\mathrm{p}<0.05)$ in ST was observed after training $(2.4 \%)$ in EG while a significant decrease $(\mathrm{p}<0.05)$ was observed $(-2.3 \%)$ in EPG. Significant increases in EPG $(p<0.05)$ were observed in SJ $(7.5 \%)$ and CMJ (7.3\%) after training, while no significant changes in both jumps were observed after training and detraining for EG. A significant increase $(\mathrm{p}<0.05)$ in MVC was observed after training $(9.1 \%)$ and after detraining $(8.1 \%)$ in EG. A significant increase $(\mathrm{p}<0.05)$ in MVC was observed after training $(16.3 \%)$. A significant increase $(\mathrm{p}<0.01)$ in CSA was observed after training in EG $(9.0 \%)$ and in EPG (7.1\%). Conclusion: EMS combined with plyometric training increased the jumping height and sprint run in physically active men. In addition, EMS alone or EMS combined with plyometric training leads to increase maximal strength and to some hypertrophy of trained muscles. However, EMS training alone did not result in any improvement in jumping explosive strength development or even may interfere in sprint run.

Key words: Maximal strength, hypertrophy, Countermovement Jump, Resistance Training 


\section{INTRODUCTION}

Paragraph Number 1. Short-term programs of electromyostimulation (EMS) are becoming recognized as an effective strategy to develop neuromuscular performance, but results are conflicting. Such studies have found that there is an increase after several weeks of EMS $(4,8,18,21,24)$, no change $(7,26,30)$ or even decrease $(25)$ in maximal voluntary strength of the lower extremity muscles, mainly during open kinetic chain efforts. These discrepancies in the results may be related to: 1) differences in EMS training protocols (i.e. number of sets and repetitions, frequency of stimulation, twitch and rest periods), 2) pretraining status of the subjects and 3) specificity of the tests to detect changes after EMS training $(11,16)$. Few studies have examined the effects of EMS-training-induced adaptations on closed kinetic explosive type of movements, such as vertical jumps. To the authors' knowledge, Maffiuleti and coworkers $(18,19)$ reported that 4 weeks of pure EMS training, or combined with plyometric training, significantly improved jumping performance in basketball and volleyball players. However, no study has evaluated the potential advantage of using EMS training during a more specific performance such as $20 \mathrm{~m}$ acceleration.

Paragraph Number 2. Coaches and researchers in the field of resistance training attempt to identify the most appropriate training stimulus that maximizes power performance enhancement, such as vertical jump or maximal acceleration. Due to the relatively high degree of task specificity involved in movement adaptation and force-velocity characteristics (15), ballistic training (23), plyometric training (1), and Olympic-style weightlifting training $(12,17)$ have resulted in significant vertical jump increases. In addition, a combined training method (e.g. ballistic training and barbell classical resistance training) has also been proven useful to enhance muscle power in a wide variety of maximal jumping, throwing and running athletic performances $(1,5,12,17,23)$. However, it remains to be seen whether EMS training protocols could be used as a complementary training method to enhance explosive athletic performance. Only one study has 
examined whether combined EMS and plyometric training had an effect on vertical jump performance (19). These authors reported that 4 weeks of combined EMS and plyometric training significantly improved jumping performance in comparison to a control group of volleyball players. All previous studies with team sports have used a time-series study design during pre-season preparation, with a volleyball training group serving as their own control. Unfortunately, no study has isolated the effects of EMS or plyometric training in order to determine the effects of combined EMS and plyometric training on explosive athletic performance. One could therefore hypothesize that combining these two methods would facilitate the improvement in maximal acceleration and jumping ability. The purpose of this study is to compare the effects of electrostimulation training alone, plyometric training alone, and electrostimulation followed by plyometric training on anaerobic power and isometric muscle strength in able-bodied individuals. A secondary aim is to estimate the CSA of the trained muscles, since there is effectively a lack of information on muscle changes following EMS training programs.

\section{METHODS}

\section{Subjects}

Paragraph Number 3. Forty male physical education students volunteered to participate in the study. Each subject gave written informed consent to participate, with the risks and benefits of the study carefully explained to them prior to its initiation. Thereafter, the subjects signed a written consent form before participation in the study. No subject performed any standardized exercise during the experimental phase. The study was conducted according to the Declaration of Helsinki and was approved by University Committee on Human Research. No subject reported any known illness or had previously experienced EMS.

\section{Experimental design}


Paragraph Number 4. The total duration of the study was 4 weeks of training. Prior to the initial testing each subject was familiarized with the testing protocol and completed a full practice testing session. After this familiarization testing session, each subject was tested on three separate occasions using identical protocols: 1) before starting training, 2) after the completion of the 4-wk training period, and 3) two weeks after the end of the training period (detraining). After baseline testing, the subjects were rank ordered by composite strength (maximal isometric strength, jump height and maximal acceleration). Thereafter, the subjects were randomly assigned to one of four treatment groups that performed 4 times per week: Electromyostimulation (EG, $\mathrm{n}=10$, age $19.4 \pm 0.4 \mathrm{yr}$; height $1.76 \pm 0.01 \mathrm{~m}$; mass $72.7 \pm 1.5 \mathrm{~kg}$ ); plyometric (PG group, $\mathrm{n}=9$, age $20.8 \pm 0.6 \mathrm{yr}$; height $1.79 \pm 0.02 \mathrm{~m}$; mass $79.7 \pm 2.1 \mathrm{~kg}$ ); combined electromyostimulation and plyometric (EPG, $\mathrm{n}=11$, age $21.4 \pm 0.9 \mathrm{yr}$; height $1.79 \pm 0.02 \mathrm{~m}$; mass $80.2 \pm 1.5 \mathrm{~kg}$ ). A control group of ten subjects did not train and were tested before and after a 4wk period to assess the reliability of the observations (CG, $n=10$, age 20.6 $\pm 0.6 \mathrm{yr}$; height $1.77 \pm 0.02 \mathrm{~m}$; mass $71.6 \pm 1.9 \mathrm{~kg})($ mean $\pm \mathrm{SD})$.

\section{Training protocols}

\section{Electromyostimulation group (EG)}

Paragraph Number 5. The EMS training of the knee extensor muscles took place four days a week for four weeks (16 sessions). Four days of training were divided by a rest day. Each session lasted $\sim 34$ min, made up of the following parts: 5 min of low frequency EMS warm up (5 Hz), and 29 min where 53 isometric contractions of both knee extensor muscles were performed. Each 3-second contraction was followed by a rest period lasting 30 seconds (rest frequency: 1Hz). The stimulator generated a biphasic symmetrical square wave signal delivered with a frequency of $120 \mathrm{~Hz}$, giving a pulse width of $400 \mu$ s $\left(\right.$ Compex $^{\circledR}$ Sport-P, Medicompex SA, Switzerland). The current level was set individually for each subject at the maximum that could be tolerated $(14,22,29)$, and the intensity tolerated levels were noted for each subject and session. 
Three self-adhesive electrodes were used on each thigh: one negative electrode $(10 \times 5 \mathrm{~cm})$ was placed on the most proximal part of the quadriceps (about $10 \mathrm{~cm}$ below the groin) (14); and two positive electrodes $(5 \times 5 \mathrm{~cm})$ were placed as close as possible to the motor point of the vastus medialis and vastus lateralis muscles (18). To standardize the knee and hip angles (Uniaxial metalic goniometer, Therapeutic Instruments ${ }^{\circledR}$, Clifton, N.J., U.S.) the subjects lay down on a mat, with their feet inside a wall bar. The knee joint was fixed at an angle of $120^{\circ}\left(180^{\circ}\right.$ corresponding to the full extension of the leg), the corresponding hip angle being constant throughout the 16 training sessions. This knee angle was used because it was the most effective in other studies $(18,29)$.

\section{Plyometric group (PG)}

Paragraph Number 6. The plyometric training took place two days a week for four weeks (8 sessions) with one rest day in between. Each session lasted 50 min, made up of the following parts: 15 min standard warm up (running for $10 \mathrm{~min}$, stretching exercises for $5 \mathrm{~min}$ and 3 submaximal sprints), $25 \mathrm{~min}$ of plyometric work, and $10 \mathrm{~min}$ of stretching exercises. The plyometric work consisted of horizontal and drop jumps. During the initial four training sessions (wk $1 \&$ 2) there were more horizontal jumps, with an average of 90 jumps per session (360 jumps). In the last four training sessions (wk $3 \& 4$ ) there were more drop jumps, with an average of 105 jumps per session (420 jumps). This distribution was chosen with the aim of increasing the intensity (from horizontal to vertical) and the volume (number of jumps) of the training sessions. To maintain maximal intensity, the recovery between two consecutive series of jumps was always complete (from 2 to $5 \mathrm{~min}$, depending on the kind and number of jumps). The training was performed on an athletics track or a synthetic floor, as these are not too hard. This is a very important aspect in plyometric training due to its high harm index.

Combined electromyostimulation and plyometric group (EPG) 
Paragraph Number 7. The 4-wk training program consisted of 16 sessions, 4 times per week: 8 EMS training sessions ( 2 each wk, similar to EG) and 8 plyometric training sessions ( 2 each wk, similar to PG). During the same week there were 2 days of EMS training and 2 days of plyometric training, with a rest day in between. PG is thus used as control group to EPG, and the benefits due to the combined training can be differentiated. The current characteristics and the electromyostimulation protocol applied to EPG were identical to those applied to EG. Likewise, the plyometric work performed by this group was similar to the PG work.

\section{Control group (CG)}

Paragraph Number 8. No training was performed by the CG. This group carried out the same testing protocols as the other groups.

\section{Testing protocols}

Paragraph Number 9. Performance testing was initiated after a standardized 10-min warm-up that included low-intensity running, several acceleration runs, jumping at a progressively increased intensity, and stretching exercises. In all neuromuscular performance tests strong verbal encouragement was given for each subject to motivate them to perform each test action as maximally and as rapidly as possible.

\section{0 m Sprint Time}

Paragraph Number 10. The sprint running tests were performed on an indoor track. The sprint running test consisted of three maximal sprints of $20-\mathrm{m}$, with a 120 -s rest period between each sprint. Running time was recorded using photocell gates (AFR Systems ${ }^{\circledR}$, AFR Technology, Spain) placed $0.4 \mathrm{~m}$ above the ground, with an accuracy of $0.001 \mathrm{~s}$. The subjects commenced the sprint when ready from a standing start, $0.5 \mathrm{~m}$ behind the start line. Stance for the start was consistent for each subject. The timer was automatically activated as the subject passed the first gate at the $0 \mathrm{~m}$ mark. In repeated determinations of sprint running time the coefficient of variation including both biological and methodological variables was less than $1.5 \%$. 
Jumping test

Paragraph Number 11. The subjects were asked to perform a maximal vertical concentric squat jump (SJ) and a maximal vertical countermovement jump with a preparatory movement from the extended leg position down to the 90-degree knee flexion, followed by a concentric action. The jumping height was calculated from the flight time. The vertical jumps were carried out on a contact mat (SportJump-v1.0 System, DSD Inc., Spain) connected to a computer (10). SJ and CMJ required the subjects to keep their hands on their waist throughout the jump. Knee flexion during the jumps was not standardized. Three maximal jumps were recorded, interspersed with approximately $10 \mathrm{~s}$ of rest, and the peak value was used for further analysis.

\section{Maximal bilateral isometric strength}

Paragraph Number 12. All subjects were tested for maximal voluntary bilateral isometric leg extension strength. The subject squatted in an isometric condition on a force platform (Dinascan $600 \mathrm{M}^{\circledR}$, Biomechanical Institute of Valencia, Spain), and was prevented from extending upwards by the shoulders being in contact with a fixed bar positioned so that the knee angle was 120 degrees. The height of the bar was maintained for each subject during the different tests. At a command a subject forcefully exerted his maximum force against the force platform by pressing his shoulders against the fixed bar. The subjects were instructed to exert their maximal force as fast as possible during a period from 2.5-4 s. The rest period between each maximal contraction was always 3 minutes. Three trials were completed for each test condition and the best performance trial was used for the subsequent statistical analysis. The maximal isometric voluntary contraction was expressed in relation to body weight (BW).

\section{Paragraph Number 13.}

\section{Thigh muscle CSA}

The circumference and skinfold of both thighs were measured in the distal section, at the electrode location middle point over the vastus lateralis and the vastus medialis. In order to 
estimate the lean tissue area (muscle and bone) the model proposed by Knapik et al. (13) was used. Thigh cross-sectional lean area (CSA) was expressed as an averaged value of the right and left thighs. All anthropometric dimensions were taken by the same tester that had previously demonstrated test-retest reliability for $\mathrm{r}>0.90$ (13). All the measurements were made three times, and the mean of the three values analyzed.

\section{Statistical Analysis}

Paragraph Number 14. Standard statistical methods were used for the calculations of the means and standard error values. One-way analysis of variance (ANOVA) was used to determine differences among the four groups' initial maximal and explosive strength, and cross-sectional lean area. Training-related effects were assessed using a two-way ANOVA with repeated measures (groups x time). When a significant F-value was achieved, Scheffe post-hoc procedures were performed to locate the pair wise differences between the means. Selected absolute changes were analyzed via one-way ANOVA. Statistical power calculations for this study ranged from 0.80 to 0.86 . The $\mathrm{p}<0.05$ criterion was used for establishing statistical significance.

\section{RESULTS}

Paragraph Number 15. No significant changes in body mass were reflected in any group through the different assessments. Moreover, no significant changes were observed in PG and CG groups in any of the variables tested at any point. Significant increases in EPG $(p<0.05)$ were observed in SJ (7.5\%) and CMJ (7.3\%) after training (Figures 1 and 2, respectively). No significant changes in SJ and CMJ were observed after training or detraining for EG. No significant differences were observed in the magnitude of the changes in SJ and CMJ after detraining between EG and EPG.

Paragraph Number 16. A significant increase $(\mathrm{p}<0.05)$ in running time was observed after training (2.4\%) in EG (Figure 3). In addition, a significant decrease $(\mathrm{p}<0.05)$ in running time was observed after training (-2.3\%) for EPG. The increase in running time after training in EG was 
greater $(\mathrm{p}<0.05)$ than that recorded in EPG. The improvements in CMJ, SJ and ST after training in EPG were greater $(p<0.05)$ than those recorded in EG.

Paragraph Number 16. A significant increase $(\mathrm{p}<0.05)$ in MVC was observed after training $(9.1 \%)$ and detraining $(8.1 \%)$ in EG (Figure 4). A significant increase $(\mathrm{p}<0.05)$ in MVC was also observed after training (16.3\%), but not after detraining in EPG. No significant differences were observed in the magnitude of the increase between EG and EPG after training in MVC. However, the increases in MVC after detraining were greater $(p<0.05)$ in $\mathrm{EG}$ than those recorded in EPG. A significant increase $(\mathrm{p}<0.01)$ in CSA was observed after training in EG $(9.0 \%)$ and in EPG (7.1\%), respectively (Figure 5). No significant differences were observed in the magnitude of the increase after training in CSA between EG and EPG.

Paragraph Number 18. Significant increases $(\mathrm{p}<0.05)$ in current intensity were performed every session in both EMS groups. During the 8 initial training sessions (from S1 to S8), no significant differences in the maximal tolerated current intensity (expressed in milliamperes) were observed among groups (Figure 6).

\section{DISCUSSION}

Paragraph Number 19. The main findings of the study indicated that a 4-week combined electromyostimulation and plyometric training program significantly increased jumping height and sprint run in physically active men. In addition, EMS alone or EMS combined with plyometric training leads to increased maximal strength and to some hypertrophy of trained muscles. However, EMS training alone did not lead to any improvement in jumping explosive strength development, or even may interfere with sprint run. These results suggest that EMS combined with plyometric training could be used to enhance athletic performance in, for example, vertical jump and maximal acceleration.

Paragraph Number 20. Few studies have examined the effects of EMS-training-induced adaptations on closed kinetic explosive type of movements such as vertical jumps and maximal 
sprint performance. In this study EPG improved the CMJ (6.7\%), SJ (7.5\%) and ST (2.3\%), while no significant change in jumping height, or even a decrease in ST, were observed in EG. Such findings are consistent with those of Maffiuletti et al $(18,19)$ and Malatesta et al $(20)$, who reported benefits on vertical jump after EMS training combined with basketball or volleyball training. However, these three studies $(18,19,20)$ did not attempt to isolate the effects of EMS training, since they also performed jumping exercises during volleyball or basketball training sessions in addition to EMS training. In the present study, because a jumping group was controlled, one could also suggest that the training-induced changes in jumping height and sprint performance could mainly be attributed to the combination of both EMS and P, and not only to their EMS program or specific type of training program (e.g. volleyball or basketball). In line with the previously reported EMS training-induced enhancement of maximal strength, it has also been shown that EMS training alone could improve the ability to perform explosive actions during isokinetic muscle actions $(4,18,21,24)$. Nevertheless, no studies have shown an optimal enhancement of EMS training alone on complex explosive type of movements such as jumps or maximal running time. This indicates that EMS training alone seems to be a positive stimulus in promoting neuromuscular enhancement during "unnatural" movements such as isokinetic and isometric testing actions and isolated specific mono-articular muscle actions such as knee extensor/flexors, but does not induce optimal stimulus during more complex neuromuscular movements. It is also likely that EMS would be delivered not only to the quadriceps muscle group but also to other important muscles participating in jumping or running (e.g. ankle and hip extensor and flexors), as well as leading to similar EMS times in explosive muscle actions (e.g. shorter EMS times without rise time) or inducing EMS during voluntary explosive actions. A positive effect on height jump could be also observed. Therefore, the effects of multiple EMS training studies (e.g. combined with other training methods or EMS alone) on athletic 
performance (e.g. jumping or throwing) warrant further investigation before being applied to high-level sportsmen.

Paragraph Number 21. Research concerning the effect of EMS training on sprint performance is very limited. After 4 weeks of EMS, the ST in EPG was significantly enhanced (2.3\%) in comparison to pretraining, whereas it remained unaltered or even decreased when $\mathrm{P}$ and EMS training were performed alone. These results contrast with the limited number of studies that have analyzed the effects of plyometric training programs on sprint performance $(6,27)$. These studies have observed that plyometric explosive training can be effective for improving shortdistance sprint performance (2.5-2.8\%) after 15-30 jumping training sessions $(6,27)$. In the present study the lack of significant changes in sprint performance after plyometric training alone may be related to an insufficient strength stimulus (i.e., two days a week for four weeks, 8 sessions), resulting in limited stimulation toward further increases in vertical jump and sprint performance, as well as to a lack of variation in the strength training program. However, as previously pointed out, combined EMS and plyometric training may be efficient enough to improve neuromuscular performance during complex and specific abilities such as sprints.

Paragraph Number 22. It is generally accepted that systematic resistance training combined with EMS can lead to considerable improvements in strength and muscle hypertrophy, when both the loading intensity and training duration are sufficient $(2,3,4,18,21,22,24,28,29)$. The present results agree with these studies, because after 4 weeks of EMS training the maximal bilateral knee strength and the lean muscle mass of the leg muscles were significantly higher in comparison to pretraining (Figure 1). Initial increases in strength and muscle mass can be greater than those recorded after a similar period of resistance training alone. Ruther et al (28) observed that increases in muscle cross-sectional area of the leg were significantly higher (10\%) after 18 EMS training sessions (3-5 sets $\times 10$ contractions $\times 1 \mathrm{~s}$ at $50 \mathrm{~Hz}$ and $500 \mu \mathrm{s})$ than those observed during a similar period of traditional voluntary resistance training program (4\%). Similarly, it has 
been previously suggested that 12-15 EMS training sessions of the quadriceps muscle group was an effective training stimulus to enhance the maximal strength of the knee and kip extensor muscles by $22 \%-44 \%(4,18,21,22,24,29)$.

Paragraph Number 23. Other studies have shown, however, that the main mechanisms responsible for EMS training-induced increases in strength could be mainly related to increases in motor unit firing frequency and maximal motor unit recruitment rates $(18,19,21)$. Thus, it would appear that the high volume EMS training (53 isometric muscle actions during $3 \mathrm{~s}$ per training session and 4 sessions per week) performed in the present study may promote positive adaptive responses for maximal strength and muscle gains as in a traditional heavy resistance training program.

Paragraph Number 24. In some instances, after highly demanding EMS training, a "rebound effect" may occur, resulting in enhanced performance when training stimulus (e.g. volume and intensity) returns to a moderate level, or even tostopping training for a short period. In the current study, training was stopped for two weeks to accomplish a "rebound effect" for all groups and avoid training volume/intensity being overreaching in nature, so that the higher volume/intensity would lead to a performance enhancement when normal training resumed over the next cycle $(9,18,19)$. To the best of the authors' knowledge, to what extent the effects of EMS training could be maintained or not has been only assessed after a short period of standardized volleyball or basketball training $(18,19,20)$ but not after stopping training completely. These studies have observed that an additional 2 weeks of volleyball or basketball training after short-term combined EMS and plyometric training could lead to a further increase in explosive strength performance $(5.5$ to $17 \%)$. In contrast to previous studies $(18,19)$, our study shows that overall explosive strength gains (e.g. height in CMJ and SJ) attained in the combined EMS and plyometric group tended to return to the pre-training values after stopping training for 2 weeks whereas, more interestingly, maximal sprint performance remained unaltered. It was 
also interesting to observe that in the EMS training alone, the overall maximal strength gains were significantly increased $(8.1 \%)$ after stopping training for a further 2 weeks. This would suggest that initial improvements in neuromuscular function may easily invoked after low volume combined EMS and plyometric training, but in contrast with high intensity EMS training programs, stopping training for a short period provides no further benefits.

Paragraph Number 25. In summary, the main findings of the study indicated that a 4-week combined electromyostimulation and plyometric training program significantly increased jumping height as well as sprint run in physically active men. In addition, EMS alone or EMS combined with plyometric training leads to increased maximal strength and to some hypertrophy of trained muscles. However, EMS training alone did not result in any improvement in jumping explosive strength development or even may interfere with sprint run. The present results suggest that EMS combined with plyometric training could be used to enhance athletic performance in abilities such as vertical jump and maximal acceleration.

\section{REFERENCES}

1. Adams K., J.P. O'Shea, K.L. O'Shea, and M Climstein. The effect of six weeks of squat, plyometric, and squat-plyometric training on power production. J. Appl. Sport Sci. Res. 6:36-41, 1992

2. Cabric, M., H.J. Appell, and A. Resic. Stereological analysis of capillaries in electrostimulated human muscles. Int. J. Sports Med. 8:327-330, 1987

3. Cabric, M., H.J. Appell, and A. Resic. Fine structural changes in electrostimulated human skeletal muscle. Eur. J. Appl. Physiol. 57:1-5, 1988

4. Colson, S., A. Martin, G. Cometti, and J. Van Hoecke. Re-examination of training by electrostimulation in human elbow musculoskeletal system. Int. J. Sports. Med. 21:281-288, 2000. 
5. Cronin J., P.J. McNair, and R.N. Marshall. Velocity Specificity, combination training and sport specific tasks. J. Sci. Med. Sport. 4:168-178, 2001

6. Diallo, O., E. Dore, P. Duche, and E. Van Praagh. Effects of plyometric training followed by a reduced training programme on physical performance in prepubescent soccer players. $J$. Sports Med. Phys. Fit. 41:342-348, 2001

7. Dooley, P., J.N. McDonagh, and M.J. White. Training using involuntary electrically evoked contractions does not increase voluntary strength. J. Physiol. 346:61, 1983

8. Duchateau, J., and K. Hainaut. Training effects of sub-maximal electrostimulation in a human muscle. Med. Sci. Sports Exer. 20:99-104, 1988

9. Fry, A.C., and W.J. Kraemer. Resistance exercise overtraining and overreaching: neuroendocrine responses. Sports Med. 23: 106-129. 1997

10. García-López, J., J. Peleteiro, J.A. Rodríguez-Marroyo, J.C. Morante, J.A. Herrero, and J.G. Villa. The validation of a new method that measures contact and flight times during vertical jump. Int. J. Sports Med. 25:1-9, 2004

11. Hainaut, K., and J. Duchateau. Neuromuscular Electrical stimulation and voluntary exercise. Sports Med. 14:100-113, 1992

12. Harris, G.R. M.H. Stone, H. S. O’Bryant et al. Short-term performance effects of high power, high force, or combined weight-training methods. J. Strength Cond. Res. 1: 14-20, 2000

13. Knapik, J.J., J.S. Staab, and E.A. Harman. Validity of an anthropometric estimate of thigh muscle cross-sectional area. Med. Sci. Sports Exerc., 28:1523:1530, 1996

14. Koutedakis, Y., R. Frischknecht, G. Vrbová, N.C. Craig Sharp, and R. Bugdett. Maximal voluntary quadriceps strength patterns in Olympic overtrained athletes. Med. Sci. Sport Exerc. 27:566-572, 1995

15. Kraemer WJ, Ratamess NA. Fundamentals of resistance training: progression and exercise prescription. Med Sci Sports Exerc. 36:674-88, 2004 
16. Lake, D.A. Neuromuscular electrical stimulation. An overview and its application in the treatment of sport injuries. Sports Med. 13:320-336, 1992

17. Lyttle, A. D., G.J. Wilson, and K.J. Ostrowsky. Enhancing performance: maximal power versus combined weights and plyometric training. J. Strength. Cond. Res. 10:173-179, 1996

18. Maffiuletti, N.A., G. Cometti, I.G. Amiridis, A. Martin, M. Pousson, and J.C. Chatard. The effects of electrostimulation training and basketball practice on muscle strength and jumping ability. Int. J. Sports Med. 21: 437-443, 2000

19. Maffiuletti N.A., S. Dugnani, M. Folz, E. Di Pierno, and F. Mauro. Effect of combined electrostimulation and plyometric training on vertical jump height. Med. Sci. Sports Exerc. 4:1638-1644, 2002

20. Malatesta, D., Cattaneo, F., Dugnani, S., Maffiuletti, N.A. Effects of electromyostimulation training and volleyball practice on jumping ability. J. Strength Cond. Res. 17: 573-579, 2003

21. Martin, L., G. Cometti, M. Pousson, and Morlon, B. The influence of electrostimulation on the mechanical and morphological characteristics of the triceps surae. J. Sports. Sci. 12:377-381, 1994

22. Miller C. and Thépaut-Mathieu C. Strength training by electrostimulation conditions for Efficacy. Int. J. Sports. Med. 14(1):20-28, 1993

23. Newton RU, W.J. Kraemer, K. Hakkinen. Effects of ballistic training on preseason preparation of elite volleyball players. Med. Sci. Sports Exerc. 31(2):323-30, 1999

24. Pichon F., J.C. Chatard, A. Martin, and G. Cometti. Electrical stimulation and swimming performance. Med. Sci. Sport Exerc. 27:1671-1676, 1995

25. Pierre, D., A.W. Taylor, M. Lavoie, W. Sellers, and Y.M. Kots. Effects of $2500 \mathrm{~Hz}$ sinusoidal current on fibre area and strength of the quadriceps femoris. J. Sports Med. 26:60-66, 1986 
26. Rich, N.C. Strength training via high frequency electrical stimulation. J. Sports Med. Phys. Fit. 32:19-25, 1992

27. Rimmer, E., and G. Sleivert. Effects of a plyometrics and conditioning research. J. Strength Cond. Res. 14:295-301, 2000

28. Ruther, C.L., Golden, C.L., Harris, R.T., Dudley, G.A. Hypertrophy, resistance training, and the nature of skeletal muscle activation. J. Strength Cond. Res. 9: 155-159, 1995

29. Selkowitz D.M. Improvement in isometric strength of the quadriceps femoris muscle after training with electrical stimulation. Phys. Ther. 65:186-196, 1985

30. Venable M.P., M.A. Collins, H.S. O’Bryant, C.R. Denegar, M.J. Sedivec and G. Alon. Effect of supplemental electrical stimulation on the development of strength, vertical jump performance and power. J. Appl. Sport. Sci. Res. 5:139-143, 1991 


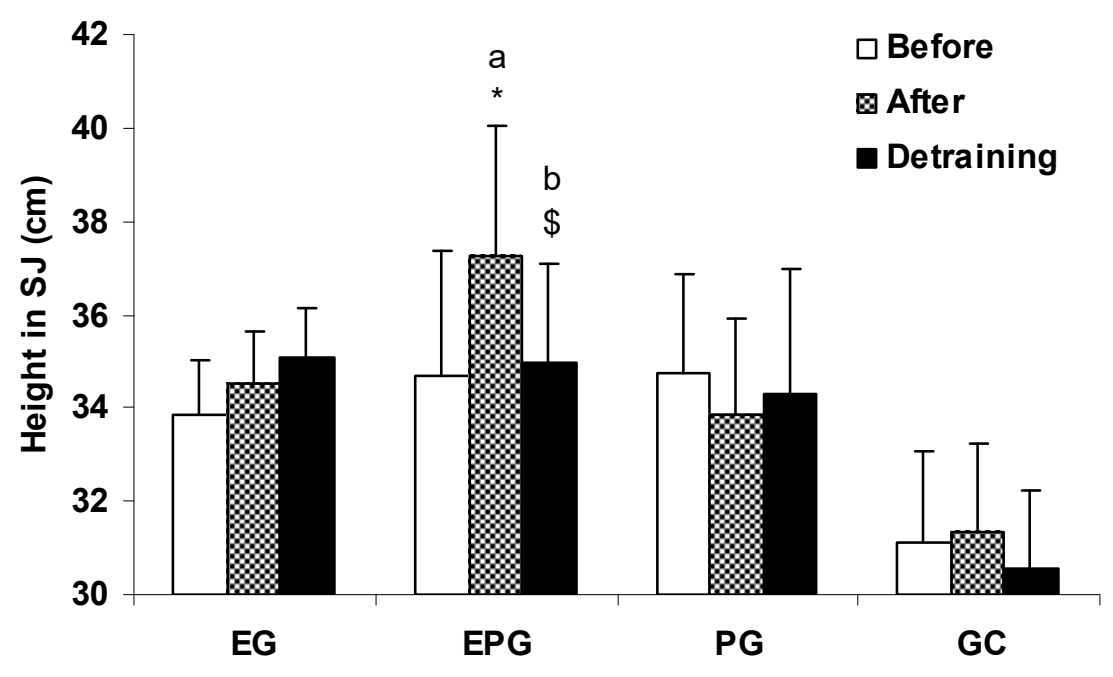

Figure 1. Vertical jump height for squat jump (SJ) in each group before and after training, as well as after 2 wk of detraining. * and \$ indicate that jump height was significantly higher than before and after training, respectively; " $a$ " indicates significant differences in the relative changes with $P G$ and $C G$ after training; *,a, $\$=p<0.05$. Values are means $\pm S E$. 


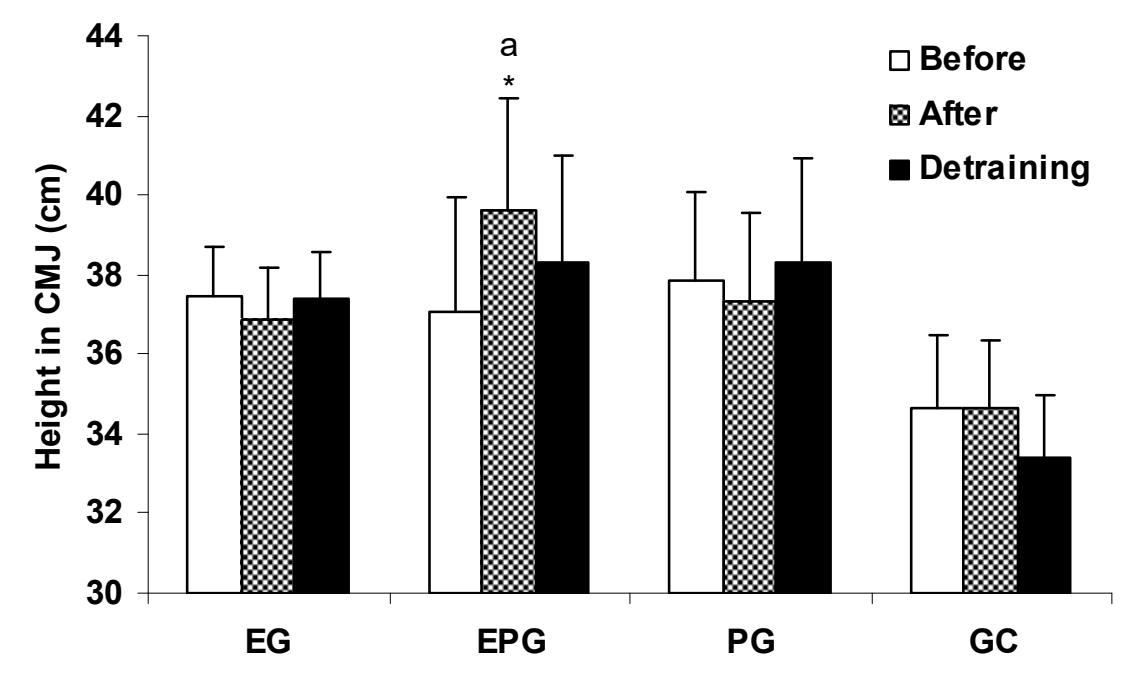

Figure 2. Vertical jump height for countermovement jump (CMJ) in each group before and after training, as well as after 2 wk of detraining. * indicates that jump height was significantly higher than before and after training, respectively; " $a$ " indicates significant differences in the relative changes with $E G$ and $P G$ after training; *,$a=p<0.05$. Values are means $\pm S E$. 


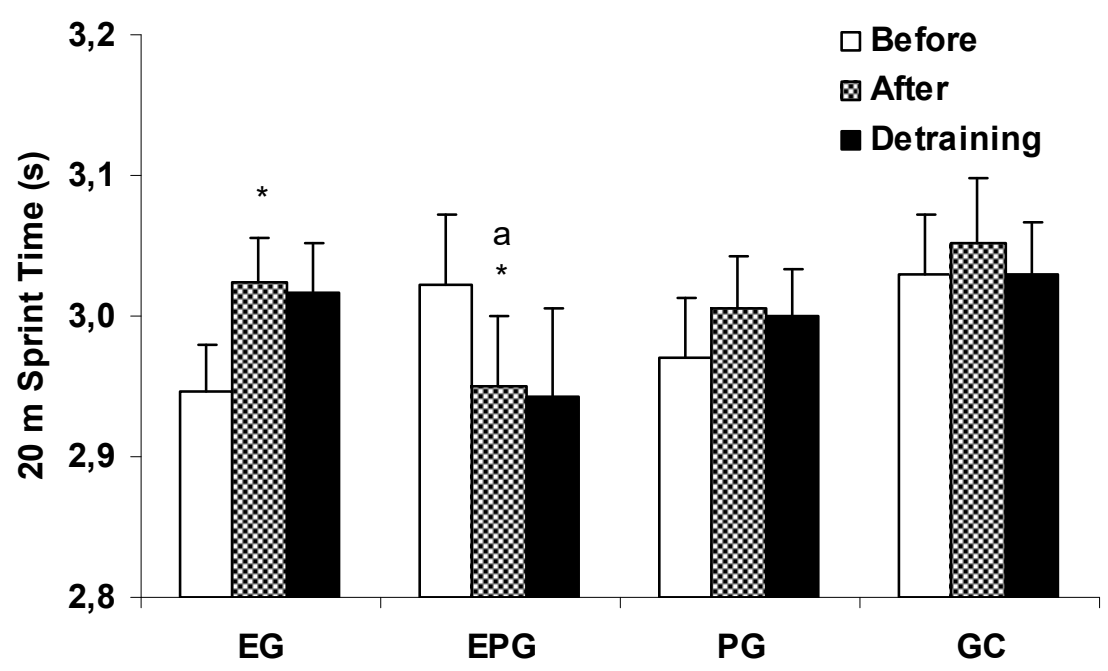

Figure 3. $20 \mathrm{~m}$ sprint time in each group before and after training, as well as after 2 wk of detraining. * indicates that ST was significantly different than before and after training, respectively; " $a$ " indicates significant differences in the relative changes with $E G, P G$ and $C G$ after training; $*, a=p<0.05$. Values are means $\pm S E$. 


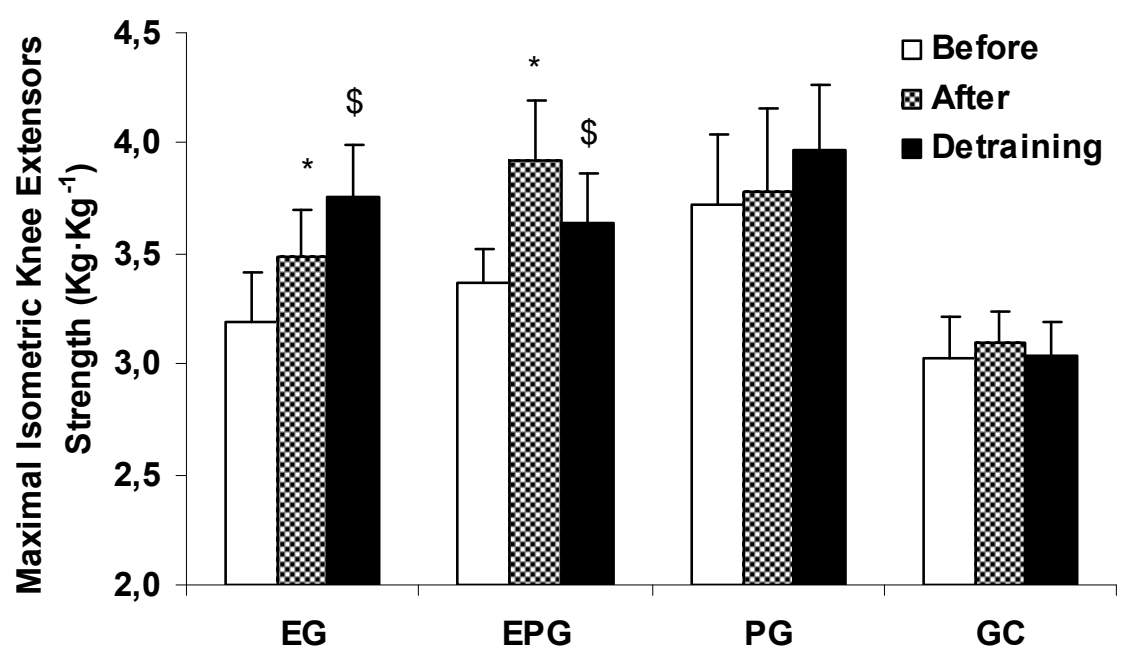

Figure 4. Knee extensors maximal isometric voluntary contraction in each group before and after training, as well as after 2 wk of detraining. * and \$ indicate that MVC was significantly higher than before and after training, respectively. $*, \$=p<0.05$. Values are means $\pm S E$. 


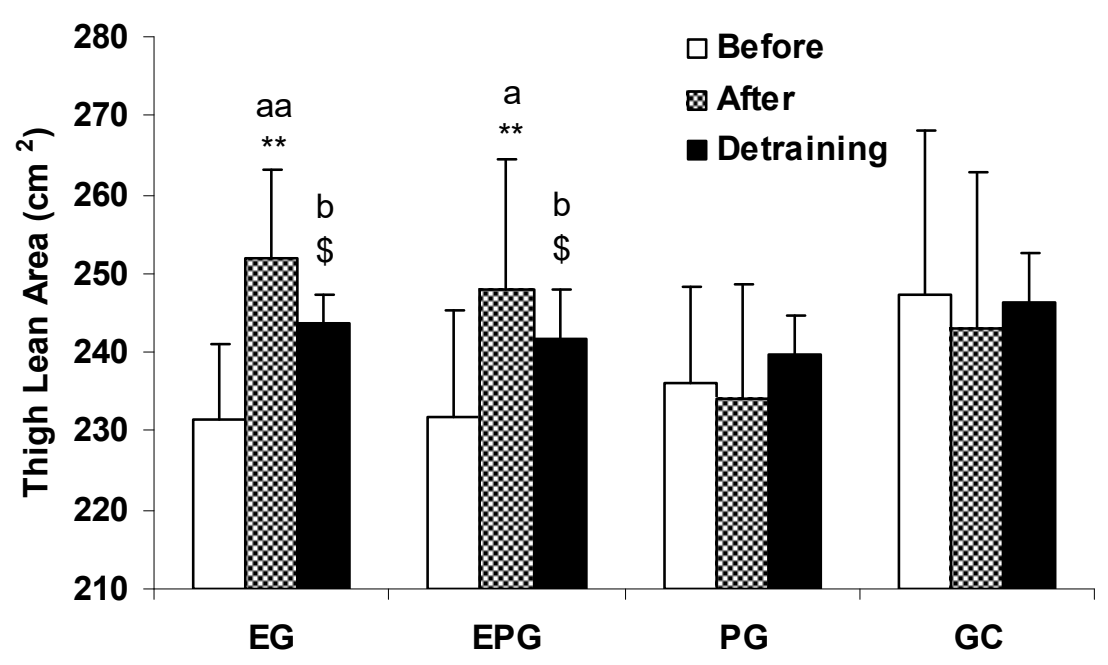

Figure 5. Thigh lean area in each group before and after training, as well as after 2 wk of detraining. * and \$ indicate that CSA was significantly higher than before and after training, respectively. " $a$ " and " $b$ " indicates significant differences in the relative changes with $P G$ and $C G$ after training and detraining, respectively. ${ }^{* *}, a a=p<0.01 ; \$, a, b=p<0.05$. Values are means $\pm S D$. 


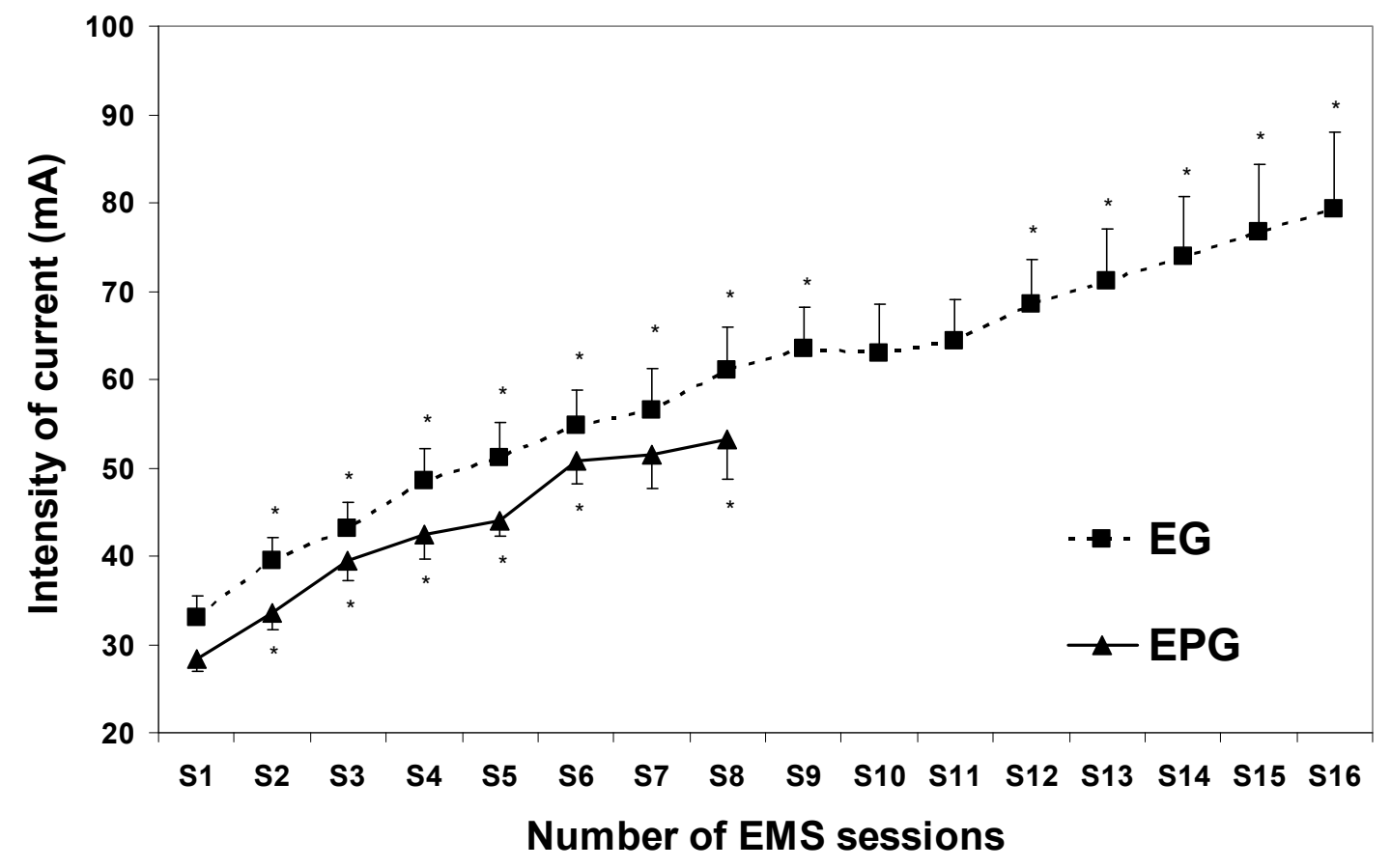

Figure 6. Current intensity mean values tolerated by EG and EPG in each EMS training sessions (S1, S2..., S16). Differences between the indicated session and the previous session. $p$ values: * $=p<0.05 . E G=$ electrostimulation group $; E P G=$ electrostimulation and plyometric group. 